\title{
INFLUÊNCIA DA PRECIPITAÇÃO NO ESCOAMENTO SUPERFICIAL EM UMA MICROBACIA HIDROGRÁFICA DO DISTRITO FEDERAL
}

\author{
DANIELLE B. S. DE ALENCAR ${ }^{1}$, CÍCERO L. DA SILVA ${ }^{2}$, CARLOS A. DA S. OLIVEIRA ${ }^{3}$
}

RESUMO: O conhecimento do escoamento superficial é importante para o dimensionamento de obras hidráulicas e para a conservação da água e do solo. O volume de água escoado superficialmente depende de fatores de natureza edafoclimática e fisiográfica da região. O objetivo deste estudo foi verificar a influência relativa do total precipitado $(\mathrm{P})$, da precipitação antecedente (PA) e da intensidade de precipitação (IP) na ocorrência de eventos de escoamento superficial (Es), na microbacia do Córrego Capetinga, situada no Centro-Oeste do Brasil, região de cerrado. Foram analisados os dados de P, PA e IP obtidos durante seis anos hidrológicos, iniciando em $1^{\circ}$ de julho de 1996 e terminando em 30 de junho 2002. Foram somente considerados valores de Es maiores ou iguais a $0,1 \mathrm{~mm}$, oriundos de hidrógrafas geradas a partir dos dados de linígrafo instalado na saída da bacia. Um conjunto de 187 dados de P, PA, IP e Es foram submetidos à análise de regressão linear múltipla. Os resultados mostraram que as variáveis independentes P, PA e IP têm dupla e tríplice interação, influenciando na variável dependente Es. Por intermédio dos valores absolutos dos coeficientes de regressão, constatou-se que IP foi mais importante do que PA na estimativa da variável Es. O valor do coeficiente de escoamento superficial total médio sugere que a microbacia possui boas condições de infiltração de água no solo.

PALAVRAS-CHAVE: recursos hídricos, cerrados, variáveis hidrológicas.

\section{PRECIPITATION INFLUENCE ON THE RUNOFF OF A SMALL-WATERSHED IN THE FEDERAL DISTRICT, BRAZIL}

\begin{abstract}
The knowledge of surface runoff is important for adequate hydraulic structures designing and for soil water conservation. The total volume of water from surface runoff is dependent of local edaphic, climatic and physiographic conditions. The objective of this study was to find out the relative importance of rainfall amount (P) and intensity (IP) and antecedent precipitation (PA) on total runoff (Es) provided by the "Capetinga" stream watershed, located in Brazil mid-west savannah region. It was analyzed watershed data for a six years period beginning on July $1^{\text {st }}, 1996$, and ending on June $30^{\text {th }}, 2002$. It was considered only Es events greater or equal to $0.1 \mathrm{~mm}$, measured with a stream gage located at the watershed outlet. A set of 187 P, PA, IP and Es events were submitted to a linear multiple regression analysis. The results showed that the independent variable P, PA and IP had two and three-factor interactions, influencing the dependent variable Es. Regression coefficients of absolute values evidenced that IP was more important than PA to estimate de independent variable Es. The average total runoff coefficient value suggests that the watershed has a good condition of soil water infiltration.
\end{abstract}

KEYWORDS: water resources, savannah region, hydrological variables.

\footnotetext{
${ }^{1}$ Mestrando, Faculdade de Agronomia e Medicina Veterinária, Universidade de Brasília, Brasília - DF, alencardani@bol.com.br.

${ }^{2}$ Professor Adjunto, Faculdade de Agronomia e Medicina Veterinária, Universidade de Brasília, Brasília - DF, cícero@unb.br.

${ }^{3}$ Professor Titular, Faculdade de Agronomia e Medicina Veterinária, Universidade de Brasília, Brasília - DF.

Recebido pelo Conselho Editorial em: 24-5-2004
}

Aprovado pelo Conselho Editorial em: 22-2-2006 


\section{INTRODUÇÃO}

A demanda mundial por água vem crescendo constantemente, contudo os suprimentos globais são limitados e sua disponibilidade vem se tornando uma questão preocupante para um número cada vez maior de países. O Brasil tem hoje uma posição privilegiada quanto ao volume de recursos hídricos, pois possui cerca de $13,5 \%$ da disponibilidade hídrica superficial mundial. Porém, mais de $73 \%$ dessa água doce encontra-se na bacia Amazônica, habitada por menos de 5\% da população. Portanto, apenas $27 \%$ dos recursos hídricos brasileiros estão disponíveis para $95 \%$ da população (FREITAS \& SANTOS, 1999).

O Distrito Federal é considerado o terceiro pior Estado brasileiro em disponibilidade de recursos hídricos per capita por ano (REBOUÇAS et al., 1999). Possui área de $5.814 \mathrm{~km}^{2}$, com cerca de $60 \%$ das terras "altas", colaborando para que a água absorvida pelo solo seja drenada para os rios de três importantes bacias hidrográficas: Prata, Araguaia-Tocantins e São Francisco (BAPTISTA, 1997). Essa característica, por si só, tem gerado constante preocupação com o uso de água na região.

Hoje se afiguram situações de graves conflitos ambientais quanto ao uso dos recursos hídricos em quase todas as principais bacias hidrográficas do Distrito Federal. Entre os principais problemas que têm comprometido o desenvolvimento sustentável do DF, estão: o crescimento acelerado da população e o aumento do consumo de água; a expansão da área irrigada sem estudos adequados de impacto ambiental; a contaminação das águas por agrotóxicos e por esgotos não tratados; as erosões e assoreamentos; as queimadas e desmatamentos; a construção de estradas, represas e outras obras de infra-estrutura sem os cuidados previstos em lei ou sem autorização dos órgãos competentes; os loteamentos, as invasões e a ocupação desordenada de terras rurais e áreas de preservação ambiental.

Para a manutenção do desenvolvimento regional sustentável, deve-se buscar o equilíbrio entre as ações voltadas para o crescimento econômico e a conservação do meio ambiente. À medida que a água se torna escassa em uma região, o conhecimento de parâmetros hidrológicos relacionados com o uso desse recurso hídrico passa a ter fundamental importância para o seu gerenciamento adequado, visando a minimizar as restrições e os conflitos pelo seu uso (SETTI et al., 2001).

Iniciada a precipitação pluviométrica, parte dela é interceptada pela vegetação, parte infiltra no solo e parte pode ser retida em depressões da superfície do terreno. Se a duração da chuva continuar, após o preenchimento dessas depressões, terá início o escoamento superficial propriamente dito. Assim, a água que escoa sob a superfície do solo, sem infiltrar, formará a enxurrada que irá compor, junto com o escoamento de base, os córregos, ribeirões, rios, lagos e reservatórios (CHOW et al., 1988).

O volume total de água transportado pelo canal de um curso de água é formado pelo escoamento superficial e pelo afluxo de água do subsolo, ou escoamento de base. No entanto, o escoamento superficial resultante das precipitações é considerado o componente preponderante na formação de cheias ou aumento de vazões dos cursos de água (PINTO et al., 1973).

A variação da vazão de um curso de água, decorrente de precipitação ocorrida na bacia de contribuição correspondente, pode ser avaliada, por exemplo, por meio de aparelhos apropriados, como os linígrafos, que registram as alturas das lâminas de água no decorrer do tempo (TUCCI, 2002). $\mathrm{O}$ conhecimento dessa grandeza interessa, sobretudo, pela possibilidade de ser correlacionada à vazão de um curso de água, por intermédio da curva-chave.

A curva-chave de um curso de água é uma função que descreve a relação entre a vazão e a altura ou cota de escoamento, levando em conta as características geométricas e hidráulicas da seção transversal do curso de água considerado (JACON \& CUDO, 1989). 
A hidrógrafa obtida a partir da curva-chave do curso de água retrata as flutuações de vazão em função do tempo (GARCEZ \& ALVAREZ, 1988) e possibilita separar o escoamento superficial do escoamento de base. Assim, o conhecimento do escoamento superficial está relacionado à identificação das disponibilidades e potencialidades hídricas de uma bacia hidrográfica.

O volume de água decorrente do escoamento superficial depende de fatores de natureza geológica, climática e fisiográfica da região (PENMAN, 1963), tais como: a área da bacia hidrográfica, a existência de declividades acentuadas e depressões retentoras de água, o tipo e o teor de água do solo, a cobertura vegetal e a quantidade e a intensidade de precipitação, entre outros.

CASTILHO et al. (1999) verificaram que a quantidade de precipitação interceptada pela cobertura vegetal, constituída predominantemente de cana-de-açúcar, em uma grande bacia hidrográfica do Estado de São Paulo, é bastante expressiva, implicando redução da parcela de escoamento superficial representada no balanço hídrico dessa bacia.

ISTOK \& BOERSMA (1986) realizaram estudo com dados de chuva e de escoamento superficial para determinar a significância relativa das chuvas antecedentes no escoamento superficial em microbacias agrícolas do Norte dos Estados Unidos, durante eventos de chuvas de baixa intensidade (de 5 a $15 \mathrm{~mm} \mathrm{~h}^{-1}$ ), concluindo que a umidade antecedente teve maior contribuição efetiva na quantidade de água escoada que a intensidade da precipitação.

FLANANGAN et al. (1988) pesquisaram, por intermédio de um simulador de chuvas, o efeito de várias intensidades de precipitação na infiltração, no escoamento superficial e na erosão, tendo constatado que os resultados obtidos foram influenciados pelas umidades iniciais presentes nos solos que receberam as precipitações artificiais.

Procurando gerar informações técnicas que possam contribuir, direta ou indiretamente, com o gerenciamento dos recursos hídricos, este estudo foi realizado no período hidrológico 1996-1997 a 2001-2002, com objetivo de: (a) quantificar os escoamentos superficiais ocorridos em uma microbacia sob vegetação natural de cerrado; (b) verificar a influência do total precipitado, da umidade antecedente e da intensidade de precipitação na ocorrência do escoamento superficial na microbacia, e (c) estabelecer a relação entre precipitação e escoamento superficial para os eventos de cheias observadas.

\section{MATERIAL E MÉTODOS}

O presente estudo foi realizado na região Centro-Oeste do Brasil, na microbacia do Córrego Capetinga, a sudeste do Distrito Federal, com centro geográfico aproximadamente no ponto $47^{\circ} 56^{\prime}$ de longitude oeste e $15^{\circ} 27^{\prime}$ de latitude sul. A microbacia possui área total de $9,62 \mathrm{~km}^{2}$, delimitada por um perímetro de $12 \mathrm{~km}$ e posicionada entre 1.057 e $1.200 \mathrm{~m}$ de altitude. A descrição da cobertura vegetal e dos diversos tipos de solo da microbacia pode ser vista em SILVA \& OLIVEIRA (1999).

O Córrego Capetinga estende-se por 2,81 km ao longo da microbacia, a qual se encontra inserida na bacia hidrográfica do Rio Paraná. A rede de drenagem da microbacia apresenta aproximadamente $18,19 \mathrm{~km}$, resultando numa densidade de drenagem igual a $1,89 \mathrm{~km} \mathrm{~km}^{-2}$. A declividade média da área é de 3,3\%, enquanto a declividade média do leito do córrego é de 1,8\%. Já o coeficiente de compacidade da microbacia $(\mathrm{Kc})$ foi determinado em 1,09. As vazões observadas no córrego, no período estudado, foram de $15,606 \mathrm{~m}^{3} \mathrm{~s}^{-1}$ para a máxima instantânea, de $0,167 \mathrm{~m}^{3} \mathrm{~s}^{-1}$ para a média e de $0,027 \mathrm{~m}^{3} \mathrm{~s}^{-1}$ para mínima-média, com sete dias de duração.

O clima na região do Distrito Federal enquadra-se entre os tipos tropical de savana e temperado chuvoso de inverno seco, caracterizado pela existência bem nítida de duas estações: uma chuvosa e quente, entre outubro e abril, e outra fria e seca, entre maio e setembro. 
Durante o período de seis anos hidrológicos consecutivos, iniciado em $1^{\underline{0}}$ de julho de 1996 e terminado em 30 de junho de 2002, foram avaliados, individualmente, todos os eventos chuvosos que ocasionaram escoamentos superficiais maiores que $0,1 \mathrm{~mm}$ na microbacia.

Em cada evento de cheia verificada, as seguintes variáveis hidrológicas foram determinadas: precipitação média na área de drenagem $(\mathrm{P})$, precipitação antecedente nos últimos cinco dias (PA), intensidade de precipitação média (IP) e escoamento superficial (Es).

Para a obtenção da precipitação média, utilizou-se do método de Thiesen e dos dados de precipitação de quatro pluviógrafos distribuídos na microbacia. As precipitações antecedentes foram determinadas com base na soma da precipitação dos cinco dias antecedentes ao dia em que ocorreu cada uma das cheias estudadas, conforme sugestão do Soil Conservation Service - SCS, United States Department of Agriculture - USDA. As precipitações antecedentes médias foram determinadas pela soma da precipitação média dos cinco dias antecedentes à chuva que provocou o evento de cheia estudado. A intensidade de precipitação média foi determinada pela divisão da precipitação média total pelo respectivo tempo médio de duração da chuva.

As elevações dos níveis da água ou cotas foram registradas pelo linígrafo situado no ponto de saída de água da microbacia. Os níveis da água no linígrafo foram coletados continuamente durante os seis anos hidrológicos abrangidos, sendo transformados em vazão do córrego, utilizando a curva-chave da estação, de controle fixo (concreto), apresentada por SILVA \& OLIVEIRA (1999).

Os dados de vazão foram correlacionados com o tempo, proporcionando a geração das hidrógrafas para cada evento de chuva correspondente. A partir dessas hidrógrafas, procederam-se as separações gráficas entre os escoamentos superficiais (Es) e os escoamentos basais (Eb), por meio do método da linha reta proposto por CHOW et al. (1988).

O volume de água escoado no córrego durante uma cheia foi obtido pela seguinte equação:

$$
\mathrm{V}=\int_{\text {to }}^{\mathrm{tn}} \mathrm{Q}(\mathrm{t}) \mathrm{dt}
$$

em que,

$\mathrm{V}$ - volume de água escoado correspondente a cada chuva, $\mathrm{m}^{3}$;

to - início do escoamento superficial, s;

tn - fim do escoamento superficial, s, e

$\mathrm{Q}(\mathrm{t})$ - vazão em função do tempo, $\mathrm{m}^{3} \mathrm{~s}^{-1}$.

Considerando que o volume total escoado é resultante da soma dos volumes de escoamento superficial e de base, obtém-se a expressão:

$$
\mathrm{Ves}+\mathrm{Veb}=\int_{\mathrm{to}}^{\mathrm{tn}}[\mathrm{Qes}(\mathrm{t})+\mathrm{Qeb}(\mathrm{t})] \mathrm{dt}
$$

em que,

Ves - volume de escoamento superficial, $\mathrm{m}^{3}$;

Veb - volume de escoamento de base, $\mathrm{m}^{3}$;

Qes(t) - vazão correspondente ao escoamento superficial em função do tempo, $\mathrm{m}^{3} \mathrm{~s}^{-1}$;

$\mathrm{Qeb}(\mathrm{t})$ - vazão correspondente ao escoamento de base em função do tempo, $\mathrm{m}^{3} \mathrm{~s}^{-1}$, e

$\mathrm{dt}$ - intervalo infinitesimal de tempo, $\mathrm{s}$.

A solução da equação anterior foi feita por métodos numéricos, com intervalos de tempo de 15 minutos e uma hora, respectivamente, para hidrógrafas de grandes e pequenas declividades. 
Para checar a influência da precipitação (P), da precipitação antecedente (PA) e da intensidade (IP), na ocorrência dos escoamentos superficiais estudados, os dados de cada uma dessas variáveis, determinados no período, foram submetidos à análise de regressão linear múltipla, estabelecendo relação entre as variáveis explicativas e a variável-resposta escoamento superficial ajustado (Ea), conforme o modelo descrito pela eq.(3). As variáveis explicativas foram P, PA, IP e suas interações.

$$
\mathrm{Ea}=\mathrm{b}_{\mathrm{o}}+\mathrm{b}_{1}(\mathrm{P})+\mathrm{b}_{2}(\mathrm{IP})+\mathrm{b}_{3}(\mathrm{PA})+\mathrm{b}_{4}(\mathrm{P} \times \mathrm{PA})+\mathrm{b}_{5}(\mathrm{P} \times \mathrm{IP})+\mathrm{b}_{6}(\mathrm{IP} \times \mathrm{PA})+\mathrm{b}_{7}(\mathrm{P} \times \mathrm{PA} \times \mathrm{IP})+\mathrm{E}
$$
em que,

$\mathrm{b}_{\mathrm{o}}$ - intercepto;

$b_{\mathrm{k}}($ para $\mathrm{k}=1,2,3,4,5,6,7)$ - coeficientes de regressão que indicam a variação de Ea com o aumento unitário da respectiva variável explicativa ou interações destas, e

E - erro aleatório devido a fatores desconhecidos.

A análise de regressão múltipla foi efetuada por meio de um programa estatístico denominado MSTAT-C. O teste " $t$ " foi utilizado para indicar quais das variáveis explicativas ou interações entre essas influenciaram na variável-resposta.

\section{RESULTADOS E DISCUSSÃO}

Entre todas as chuvas registradas no período dos seis anos hidrológicos estudados, destacaram-se o total de 187 (Tabela 1), que resultaram em escoamentos superficiais maiores ou iguais a $0,1 \mathrm{~mm}$. Esses eventos de chuvas produziram o total de água escoado superficialmente equivalente a 292,3 mm, enquanto o somatório das precipitações responsáveis por esse escoamento foi de 4.203,3 mm para um total precipitado, no período, de $8.223,6 \mathrm{~mm}$.

Ao relacionar as maiores lâminas de escoamento superficial com os respectivos valores de precipitação, intensidades de precipitação e precipitação antecedente, foi verificado que essas lâminas não estão, necessariamente, associadas aos maiores valores dessas variáveis hidrológicas. Tal fato mostra que a ocorrência do escoamento superficial não pode ser explicada pela influência de uma única variável, embora tenha sido observado que os maiores escoamentos superficiais estejam sempre associados a valores elevados de precipitação.

No detalhamento dos escoamentos superficiais e respectivas chuvas (Tabela 1), destaca-se, por exemplo, a maior lâmina de escoamento superficial observada no período estudado, que foi de 10,2 mm, ocorrida no ano hidrológico de 1996-1997 e associada à oitava maior precipitação observada no mesmo período. Por outro lado, os maiores valores de intensidade de precipitação e de precipitação antecedente, observados no período estudado, foram, respectivamente, $43,0 \mathrm{~mm} \mathrm{~h}^{-1}$ e $138,4 \mathrm{~mm}$, ocorridos em dias diferentes e não associados às maiores lâminas de escoamento superficial, mostrando que o escoamento não pode ser explicado somente por uma única variável.

O coeficiente de escoamento superficial (C), razão entre a lâmina escoada e a respectiva precipitação, foi calculado para os 187 eventos de chuvas selecionados, sendo o maior deles de 0,318. Diferentes classes de $C$ podem ser visualizadas na Tabela 2, cujo valor médio é de 0,069 . No entanto, quando se divide a lâmina total de escoamento superficial pela precipitação total dos anos hidrológicos estudados, chega-se a um valor médio de 0,036 . Os pequenos valores dos coeficientes de escoamentos superficiais da microbacia estão associados às características físicas do solo e à excelente cobertura desse, indicando boas condições de infiltração. 
TABELA 1. Dados de precipitação (P, mm), intensidade de precipitação (IP, $\mathrm{mm}$ ), precipitação antecedente (PA, mm), escoamento superficial observado (Es, $\mathrm{mm}$ ) e ajustado (Ea, $\mathrm{mm}$ ) para a bacia do Córrego Capetinga, Brasília - DF, nos seis anos hidrológicos estudados.

\begin{tabular}{|c|c|c|c|c|c|c|c|c|c|c|c|c|c|c|}
\hline \multicolumn{5}{|c|}{ 1996-1997 } & \multicolumn{5}{|c|}{ 1997-1998 } & \multicolumn{5}{|c|}{ 1998-1999 } \\
\hline $\mathrm{P}$ & IP & PA & Es & $\mathrm{Ea}$ & $\mathrm{P}$ & IP & PA & Es & $\mathrm{Ea}$ & $\mathrm{P}$ & IP & PA & Es & $\mathrm{Ea}$ \\
\hline 19,8 & 4,4 & 79,0 & 0,2 & 1,7 & 29,5 & 10,9 & 25,4 & 0,5 & 2,2 & 35,6 & 6,0 & 56,0 & 2,0 & 2,9 \\
\hline 12,0 & 1,0 & 85,0 & 0,2 & 0,9 & 22,2 & 4,8 & 54,2 & 0,6 & 1,7 & 33,2 & 1,8 & 61,0 & 1,5 & 0,2 \\
\hline 11,6 & 2,0 & 14,0 & 0,1 & 0,4 & 19,9 & 27,2 & 29,8 & 0,7 & 1,4 & 35,2 & 4,3 & 51,7 & 3,2 & 3,2 \\
\hline 30,0 & 3,4 & 12,0 & 0,6 & 1,9 & 19,6 & 3,9 & 37,6 & 1,3 & 1,3 & 8,4 & 8,4 & 14,5 & 2,0 & 1,0 \\
\hline 16,4 & 9,5 & 42,0 & 0,1 & 1,0 & 24,1 & 5,2 & 32,7 & 0,8 & 1,6 & 39,1 & 16,0 & 16,7 & 1,7 & 0,0 \\
\hline 37,7 & 17,2 & 91,0 & 8,2 & 2,5 & 18,3 & 2,7 & 33,9 & 0,4 & 1,1 & 14,0 & 0,8 & 62,6 & 0,5 & 1,4 \\
\hline 14,2 & 7,1 & 24,0 & 0,3 & 0,7 & 94,4 & 15,8 & 18,8 & 6,2 & 8,4 & 2,4 & 2,7 & 53,7 & 0,3 & 0,3 \\
\hline 16,5 & 3,2 & 46,0 & 0,6 & 1,1 & 24,3 & 6,8 & 107,6 & 0,4 & 2,2 & 19,7 & 15,1 & 9,0 & 0,3 & 0,7 \\
\hline 27,9 & 3,8 & 67,0 & 0,2 & 2,4 & 5,3 & 5,3 & 59,1 & 0,5 & 0,0 & 8,8 & 0,4 & 32,4 & 0,1 & 2,0 \\
\hline 63,5 & 5,2 & 24,0 & 10,2 & 5,0 & 13,0 & 3,8 & 48,3 & 0,4 & 0,7 & 13,8 & 6,1 & 32,2 & 0,5 & 0,2 \\
\hline 10,3 & 3,0 & 82,0 & 0,2 & 0,7 & 3,1 & 0,4 & 34,5 & 0,1 & 0,0 & 30,4 & 2,6 & 22,1 & 0,1 & 2,4 \\
\hline 20,6 & 3,2 & 21,0 & 0,2 & 1,2 & 32,5 & 26,6 & 1,0 & 2,3 & 3,3 & 6,9 & 2,1 & 56,0 & 0,1 & 0,2 \\
\hline 19,8 & 15,6 & 32,0 & 0,2 & 1,3 & 33,8 & 24,6 & 36,6 & 2,7 & 2,6 & 29,0 & 3,2 & 55,7 & 1,0 & 0,8 \\
\hline 22,9 & 4,0 & 26,0 & 0,3 & 1,5 & 97,2 & 18,2 & 72,4 & 3,5 & 7,5 & 7,4 & 2,3 & 52,9 & 0,1 & 1,9 \\
\hline 21,4 & 2,2 & 28,0 & 1,3 & 1,3 & 9,7 & 6,4 & 119,7 & 1,0 & 0,6 & 14,8 & 8,5 & 10,2 & 0,3 & 1,4 \\
\hline 35,6 & 6,1 & 52,0 & 3,8 & 2,9 & 32,2 & 10,7 & 96,0 & 1,1 & 2,6 & 27,9 & 5,3 & 24,0 & 0,7 & 1,6 \\
\hline 28,4 & 8,3 & 53,0 & 2,7 & 2,2 & 37,5 & 7,3 & 128,0 & 2,6 & 3,9 & 17,7 & 2,2 & 69,6 & 0,1 & 0,8 \\
\hline 15,2 & 8,8 & 42,0 & 0,2 & 0,9 & 16,6 & 4,4 & 74,0 & 0,2 & 1,3 & 17,9 & 1,0 & 86,2 & 0,7 & 4,6 \\
\hline 44,7 & 9,5 & 31,0 & 4,0 & 3,5 & 85,4 & 15,2 & 58,0 & 10,0 & 7,1 & & & & & \\
\hline 15,2 & 6,1 & 68,0 & 1,0 & 1,0 & 11,8 & 3,0 & 102,2 & 0,8 & 0,9 & & & & & \\
\hline 15,8 & 5,1 & 4,0 & 0,8 & 0,7 & 27,4 & 3,9 & 105,3 & 3,5 & 2,8 & & & & & \\
\hline 45,8 & 5,0 & 59,0 & 6,6 & 4,1 & 19,4 & 5,6 & 15,8 & 0,3 & 1,1 & & & & & \\
\hline 18,7 & 3,0 & 76,0 & 1,1 & 1,6 & 47,3 & 19,0 & 37,0 & 4,9 & 3,8 & & & & & \\
\hline 9,1 & 1,1 & 104,0 & 0,2 & 0,7 & 15,4 & 4,5 & 104,4 & 0,4 & 1,3 & & & & & \\
\hline 40,7 & 1,2 & 9,0 & 2,7 & 2,5 & 19,9 & 2,5 & 107,3 & 1,3 & 2,0 & & & & & \\
\hline 21,2 & 8,2 & 51,0 & 0,2 & 1,5 & 8,5 & 8,0 & 107,7 & 1,4 & 0,4 & & & & & \\
\hline 31,8 & 4,9 & 38,0 & 2,9 & 2,4 & 10,1 & 4,1 & 47,0 & 1,0 & 0,5 & & & & & \\
\hline 31,8 & 6,4 & 0,0 & 0,4 & 2,0 & 17,4 & 13,8 & 18,6 & 1,2 & 1,1 & & & & & \\
\hline 42,2 & 19,8 & 32,0 & 0,6 & 3,4 & 8,7 & 2,2 & 36,7 & 0,7 & 0,3 & & & & & \\
\hline 15,4 & 1,5 & 74,0 & 0,2 & 1,2 & 20,2 & 5,8 & 39,0 & 1,0 & 1,3 & & & & & \\
\hline 25,7 & 9,2 & 0,0 & 0,7 & 1,7 & 10,3 & 10,3 & 52,1 & 0,3 & 0,5 & & & & & \\
\hline & & & & & 25,8 & 43,0 & 11,4 & 1,1 & 2,9 & & & & & \\
\hline & & & & & 17,8 & 6,8 & 0,0 & 0,1 & 0,9 & & & & & \\
\hline & & & & & 13,1 & 3,0 & 17,8 & 0,3 & 3,0 & & & & & \\
\hline
\end{tabular}

continua... 
TABELA 1. Continuação...

\begin{tabular}{|c|c|c|c|c|c|c|c|c|c|c|c|c|c|c|}
\hline \multicolumn{5}{|c|}{ 1999-2000 } & \multicolumn{5}{|c|}{ 2000-2001 } & \multicolumn{5}{|c|}{$2001-2002$} \\
\hline $\mathrm{P}$ & IP & $\mathrm{PA}$ & Es & $\mathrm{Ea}$ & $\mathrm{P}$ & IP & PA & Es & $\mathrm{Ea}$ & $\mathrm{P}$ & IP & PA & Es & $\mathrm{Ea}$ \\
\hline 13,6 & 2,4 & 41,1 & 1,7 & 1,1 & 10,6 & 2,2 & 40,3 & 0,4 & 0,5 & 23,9 & 6,6 & 6,6 & 0,3 & 1,4 \\
\hline 47,6 & 24,1 & 11,1 & 8,7 & 4,6 & 8,6 & 1,2 & 21,6 & 0,9 & 0,2 & 118,7 & 5,0 & 20,9 & 2 & 9,6 \\
\hline 17,5 & 77 & 31,9 & 0,6 & 1,1 & 24,5 & 1,9 & 20,9 & 0,6 & 1, & 26 & 3,1 & 18,3 & 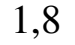 &, 7 \\
\hline 40,8 & 13,6 & 48,9 & 3,3 & 3,2 & , 0 & 8,2 & 44,7 & 4 & & 4, & 2,3 & 19,4 & 3 & 0,7 \\
\hline 31,7 & 7 & 42,5 & 2,9 & 2,4 & 9 & 3,0 & 63,6 & 0,9 & 0 & 8 & 5,8 & 26,8 & ,9 &, 2 \\
\hline 16,8 & 5,6 & 29,1 & 1,6 & 1,0 & 12,8 & 4,0 & 39,8 & 0,8 & 0,7 & 35,9 & 7,9 & 26,5 & 1,7 & 2,7 \\
\hline 30,7 & 4,4 & 55,4 & 2,7 & 2,5 & 8,4 & 1,4 & 29,2 & 1,1 & 0,2 & 3,7 & 2,4 & 14,1 & 0,4 & 0,0 \\
\hline 8,2 & 2,3 & 11,6 & 0,3 & 0,1 & 13,5 & 13,5 & 1,5 & 0,8 & 0,7 & 16,3 & 4,5 & 0,0 & 0,5 & 0,7 \\
\hline 18,7 & 1,6 & 31,4 & 0,5 & 1,1 & & & 15,0 & 4 & & 9, & 2,9 & 16,9 & 4 & 0,3 \\
\hline 14,1 & 2, & 33,9 & 1,2 & 0,8 & 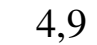 & , 1 & 41,1 & 3 & 0 & & 4,2 & 13,9 & 0,7 & 0,5 \\
\hline 19,1 & 3,1 & 5,3 & 0,5 & 0,9 & 73,3 & 18,0 & 12,7 & 9,0 & 6 & 19 & 5,0 & 16,5 & 4,9 & 1,1 \\
\hline 13,0 & 11,6 & 12,0 & 0,2 & 0,7 & 40,1 & 4,2 & 94,0 & 2,8 & 4,1 & 15,6 & 3,1 & 36,9 & 1,0 & 0,9 \\
\hline 26,0 & & 38,2 & 0,9 & 1,9 & 13,1 & 2,7 & 4,0 & 0,2 & 0,5 & 7,9 & 3,0 & 27,9 & 8 & 0,2 \\
\hline 9,8 & 4, & 43,6 & 0,4 & 0,4 & & 2 & 1,4 & 0 & 0 & 2 & 2,1 & 38,1 & 1,8 & 1,6 \\
\hline 24,2 & 5 & $6 /, 2$ & 1 & 0, & & & 29,3 & & 0 & & 4, & 0,0 & 2 & 0,4 \\
\hline 8,9 & 2 & 43,2 & 0,4 & 0,3 & 17,0 & 9,5 & 4,6 & 0,3 & 1 & 23 & 3,7 & 15,5 &, 7 & 1,4 \\
\hline 10,9 & 3, & 67,2 & 0,5 & 0,6 & & 9,1 & 24,0 & 0,6 & 2 , & 11 & 3,6 & 38,6 & 0,5 & 0,6 \\
\hline 13,7 & 7 , & 43,5 & 0,6 & 0,8 & & 4,7 & 6,4 & 0,4 & 0 & 7 & 0,9 & 23,1 & 0,1 & 0,1 \\
\hline 7,5 & 4, & 13 & 0,2 & 0,1 & 0 & 10 & 5,1 & 1 , & 2 & 21 & 4 & 30,0 & 1,2 & 1,4 \\
\hline 16,8 & 9 & 33,0 & 1 , & 1, & 10 & 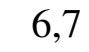 & 31,0 & 0 & U & & 3, & 49,7 & 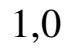 & 0,9 \\
\hline 29,2 & 11,0 & 7,4 & 0,7 & 2,1 & & 2,4 & 42,0 & 0,1 & 0 & 28,9 & 7,8 & 65,6 & 2,7 & 2,3 \\
\hline 65,4 & & 13,5 & 3,1 & 4,9 & & 7,6 & 12,1 & 0,2 & 0 & & 4,2 & 6,0 & ), 1 & 0,0 \\
\hline \multirow[t]{4}{*}{9,2} & 3,6 & 12,5 & 0,9 & 0,2 & 10,9 & 8,0 & 22,6 & 0,3 & 0 , & 17 & 4,6 & 10,2 & 0,5 & 0,9 \\
\hline & & & & & 41,2 & 5,4 & 42,4 & 7,8 & 3 & 12,7 & 2,4 & 23,2 & 0,5 & 0,5 \\
\hline & & & & & 11 , & 1,3 & 58,1 & 0,8 & 0 & 27,0 & 5,4 & 43,2 & 3,7 & 2,0 \\
\hline & \multicolumn{4}{|c|}{ 00-2001 (início) } & 3,9 & 0,6 & 67,9 & 0,6 & 0,0 & 10,7 & 4,6 & $-0,1$ & 3,4 & 2,5 \\
\hline 22,7 & 3 , & 21,4 & 0,5 & 1,4 & 29,3 & 4,5 & 71,8 & 3,9 & 5 , & 29,6 & 3,9 & 46,7 & 3,0 & 2,3 \\
\hline 11,5 & 5, & 44,1 & 0,6 & 0,6 & & 1,3 & 51,0 & 0,6 & 0 & 8,0 & 1,9 & 76,2 & 0,4 & 0,4 \\
\hline 19,3 & 4,1 & 8,3 & 0,5 & 1,0 & & 4,2 & 56,4 & 5 , & 3 & 13,2 & 5,5 & 84,2 & 1,1 & 0,9 \\
\hline 76,3 & 7,6 & 27,6 & 3,8 & 6,3 & & 1,8 & 91,4 & 2 & 1, & 3,2 & 3,0 & 98,1 & 0,8 & 0,0 \\
\hline 41,2 & 5,5 & 102,3 & 6,2 & 4,3 & & 2,4 & 7,8 & 0,8 & 0 & 11,7 & 8,0 & 65,2 & 1,1 & 0,6 \\
\hline 16,5 & 2,2 & 138,4 & 0,6 & 1,9 & & 7,1 & 7,1 & 1,1 & 1, & 40,7 & 13,6 & 39,5 & 7,4 & 3,2 \\
\hline 2,9 & 2, & 73,2 & 0,2 & 0,0 & & 6,5 & 35,8 & 2,7 & 1 , & 28,2 & 1,2 & 58,1 & 3,1 & 2,4 \\
\hline 12,6 & 34,3 & 2,3 & 0,2 & 1 , & 23 & 7,5 & 32,4 & 3,7 & 1, & 38,4 & 3,5 & 85,7 & 6,2 & 3,9 \\
\hline \multirow[t]{2}{*}{22,2} & 2,6 & 20,9 & 0,6 & 1,3 & 14 & 3,6 & 6,5 & 0,5 & 0 , & 31,2 & 5,7 & 1,7 & 0,8 & 1,9 \\
\hline & & & & & 36,9 & 10,1 & 0,0 & 2,9 & 2,7 & 32,3 & 10,8 & 32,8 & 1,9 & 2,4 \\
\hline
\end{tabular}

TABELA 2. Intervalo de classes de coeficientes de escoamentos superficiais "C" observados na Bacia do Córrego Capetinga.

\begin{tabular}{ccc}
\hline Classe de "C" & Eventos & $\%$ \\
\hline $0,000-0,049$ & 94 & 50,27 \\
$0,050-0,099$ & 57 & 30,48 \\
$0,100-0,199$ & 31 & 16,58 \\
$>0,200$ & 5 & 2,67 \\
\hline
\end{tabular}


O teste "t" revelou que todas as hipóteses nulas de que as variáveis hidrológicas P, PA e IP e as interações entres essas não influenciam no escoamento superficial foram rejeitadas. Isso implica dizer que tanto o total precipitado $(\mathrm{P})$ como a interação desse com a intensidade de precipitação (P x IP), com a precipitação antecedente (P x PA) e ainda com PA e IP (P x PA x IP), foram significativas, ou seja, as variáveis explicativas influenciaram na variável-resposta (Tabela 3).

$\mathrm{Na}$ análise estatística empregada, constatou-se que o efeito aditivo de $\mathrm{P}$ é pouco significativo em relação às outras hipóteses testadas, razão pela qual se optou por um modelo que exclui o efeito aditivo dessa variável hidrológica no modelo adotado. No entanto, a influência de "P" no escoamento superficial aparece nas interações dessa com as demais variáveis hidrológicas testadas, confirmando que não é apenas a ocorrência de precipitação que determina a quantidade de escoamento superficial, mas também a interação dessa com outras variáveis.

A partir dos valores absolutos dos coeficientes de regressão, observou-se, no período estudado, IP com maior influência na ocorrência do escoamento superficial que PA, uma vez que $b_{2}$ foi maior que $b_{3}$, como $b_{4}$ foi maior que $b_{5}$, ou seja, tanto o efeito aditivo de IP foi maior que o efeito aditivo de PA, como o efeito da interação entre IP e P foi maior que o efeito da interação entre PA e P.

Esse resultado mostra o oposto do que foi encontrado por ISTOK \& BOERSMA (1986) que, analisando escoamentos superficiais de chuvas de intensidade de 5 a $15 \mathrm{~mm} \mathrm{~h}^{-1}$, concluíram que a precipitação antecedente a uma referida precipitação foi mais preponderante na formação do escoamento superficial do que a intensidade de precipitação. Essa contradição talvez esteja associada às diferenças entre as características físicas das bacias estudadas, além das características das precipitações que, no caso presente, apresentaram amplitude maior na variável IP $\left(0,4\right.$ a $\left.43 \mathrm{~mm} \mathrm{~h}^{-1}\right)$.

TABELA 3. Resumo do teste " $t$ " para o modelo de regressão linear múltipla adotado.

\begin{tabular}{cccc}
\hline Variáveis Explicativas & Coeficiente de Regressão & Valor de "t" & Probabilidade \\
\hline IP & $-1,836 \times 10^{-1}\left(\mathrm{~b}_{2}\right)^{*}$ & $-5,031$ & 0,000 \\
PA & $-3,439 \times 10^{-2}\left(\mathrm{~b}_{3}\right)$ & $-5,148$ & 0,000 \\
P x IP & $8,027 \times 10^{-3}\left(\mathrm{~b}_{4}\right)$ & 8,544 & 0,000 \\
P x PA & $1,993 \times 10^{-3}\left(\mathrm{~b}_{5}\right)$ & 8,363 & 0,000 \\
IP x PA & $4,892 \times 10^{-3}\left(\mathrm{~b}_{6}\right)$ & $-7,249$ & 0,000 \\
P x IP x PA & $1,864 \times 10^{-4}\left(\mathrm{~b}_{7}\right)$ & 4,382 & 0,000 \\
\hline
\end{tabular}

*o valor do coeficiente $b_{1}$ foi negligenciado em razão de a variável $P$ não ter sido significativa.

Tomando o valor do intercepto e dos coeficientes de regressão, o modelo estatístico, com $\mathrm{R}^{2}$ igual a 0,60 , ficou assim representado:

$$
\begin{aligned}
& \mathrm{Ea}=1,014-1,836 \times 10^{-1}(\mathrm{IP})-3,4390 \times 10^{-2}(\mathrm{PA})+8,027 \times 10^{-3}(\mathrm{P} \times \mathrm{IP})+1,993 \times 10^{-3} \\
& (\mathrm{P} \times \mathrm{PA})+4,892 \times 10^{-3}(\mathrm{IP} \times \mathrm{PA})+1,864 \times 10^{-4}(\mathrm{P} \times \mathrm{IP} \times \mathrm{PA})
\end{aligned}
$$

Ao correlacionar os valores de escoamento observados com os ajustados pelo modelo (Figura 1), foi verificado que o modelo estatístico obtido subestima os maiores valores de Es e superestima os menores valores de Es, pois a declividade da reta resultante foi menor que um e o intercepto maior que zero. Um coeficiente de determinação $\mathrm{R}^{2}=0,60$ indica que $60 \%$ da variabilidade dos dados foi explicada pela regressão. 


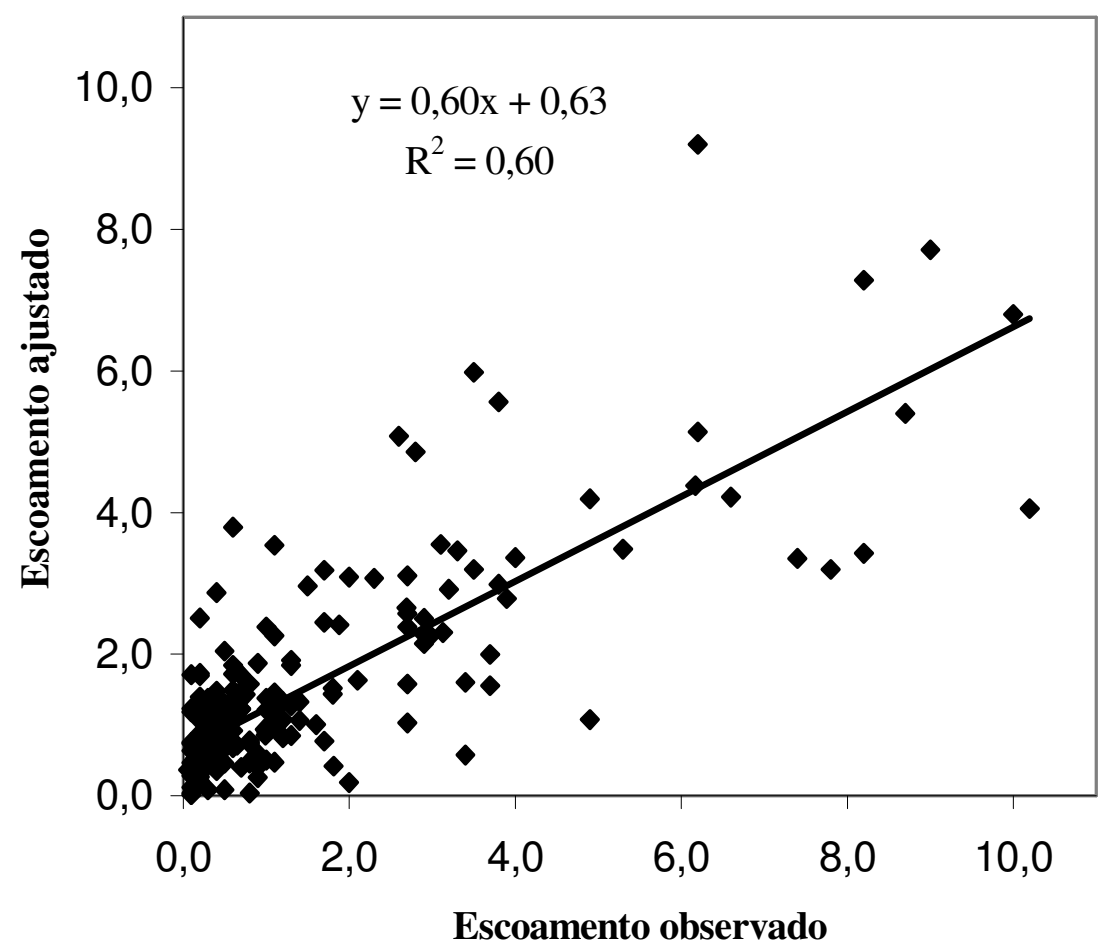

FIGURA 1. Valores de escoamento superficial observado e ajustado pelo modelo de regressão linear múltipla adotado.

\section{CONCLUSÕES}

A microbacia apresentou boas condições de infiltração de água no solo, com coeficiente de escoamento superficial médio de 0,036 .

No período hidrológico estudado, as variáveis precipitação antecedente, intensidade de precipitação e as interações entre essas e a variável precipitação, e ainda a interação entre ambas (intensidade de precipitação versus precipitação antecedente), influenciaram significativamente na ocorrência de escoamento superficial na microbacia do Córrego Capetinga.

A intensidade de precipitação teve maior influência na ocorrência de escoamento superficial ajustado do que a precipitação antecedente.

O efeito aditivo da precipitação é pouco significativo, porém sua influência no escoamento superficial ajustado aparece nas interações desta com as demais variáveis hidrológicas testadas.

\section{REFERÊNCIAS}

BAPTISTA, G.M.M. Diagnóstico ambiental e perda laminar de solos no Distrito Federal, por meio de geoprocessamento. 1997. 112 f. Dissertação (Mestrado em Geociências) - Faculdade de Agronomia e Medicina Veterinária, Universidade de Brasília, Brasília, 1997.

CASTILHO, C.P.G.; TEIXEIRA FILHO, J.; LULU, J. Interceptação de chuvas na cultura de canade-açúcar (Saccharum officinarum spp.). In: SIMPÓSIO BRASILEIRO DE RECURSOS HÍDRICOS, 8., 1999, Belo Horizonte. Resumos... Belo Horizonte: Associação Brasileira de Recursos Hídricos, 1999. p.84. 
CHOW, V.T.; MAIDMENT, D.R.; MAYS, L.W. Applied hydrology. New York: McGraw-Hill, 1988. 572 p. (Water Resources and Environmental Engineering).

FLANANGAN, D.C.; FOSTER, G.R.; MOLDENHAUER, W.C. Storm pattern effect on infiltration, runoff and erosion. Transaction of the ASAE, St. Joseph, v.31, n.2, p.11-26, 1988.

FREITAS, M.A.V.; SANTOS, A.H.M. Perspectivas de gestão e informações de recursos hídricos. $O$ Estado das Águas no Brasil. Brasília: SIH/ANEEL, MMA, SRH. 1999. 336 p.

GARCEZ, L.N.; ALVAREZ, G.A. Hidrologia. 2.ed. São Paulo: Edgar Blücher, 1988. 291 p.

ISTOK, J.D.; BOERSMA, L. Effect of antecedent rainfall on runoff during low-intensity rainfall. Journal of Hydrology, Amsterdam, v.88, p.329-42, 1986.

JACON, G.; CUDO, K.J. Curva-chave: análise e traçado. Brasília: DNAEE, 1989. 273 p.

PENMAN, H.L. Vegetation and hydrology. London: Commonwealth Agricultural Bureau, 1963. 124 p. (Technical communication, 53)

PINTO, N.L.; HOLTZ, A.C.T.; MARTINS, J.A. Hidrologia de superfície. 2.ed. São Paulo: Edgard Blücher, 1973. $179 \mathrm{p}$.

REBOUÇAS, A.C.; BRAGA, B.; TUNDISI, J.G. Águas doces no Brasil: capital ecológico, uso e conservação. São Paulo: Escrituras Editora, 1999. 717 p.

SETTI, A.S.; LIMA, J.E.F.W.; CHAVES, A.G.M; PEREIRA, I.C. Introdução ao gerenciamento de recursos hídricos. 3.ed. Brasília: Agência Nacional de Energia Elétrica; Agência Nacional das Águas, 2001. 328 p.

SILVA, C.L.; OLIVEIRA, C.A.S. Runoff measurement and prediction for a watershed under natural vegetation in central Brazil. Revista Brasileira de Ciência do Solo, Viçosa, v.23, n.3, p.695-701,1999.

TUCCI, C.E.M. Regionalização de vazões. Porto Alegre: Universidade Federal do Rio Grande do Sul, 2002. $256 \mathrm{p}$. 